\title{
Block Copolymerization. II. Polymerization of Tetrahydrofuran by Bifunctional Cationic Initiator
}

\author{
Yuya Yamashita, Masahiro Hirota, Hisashi Matsui, \\ Akira Hirao, and Koji Nobutoki \\ Department of Synthetic Chemistry, Faculty of Engineering, \\ Nagoya University, Nagoya, Japan.
}

(Received August 20, 1970)

\begin{abstract}
Polymerization of tetrahydrofuran (THF) by 2-methyl-1,3-dioxolenium perchlorate clarified the living nature of the polymerization. Although the initiation reaction is slower than the propagation reaction, the bonding mechanism of the initiation was shown from NMR and IR, and the formation of one polymer chain from one catalyst molecule was substantiated. Poly-THF di-cation of $\overline{M_{n}} 173000$ to 322000 was prepared in high vacuum system by using a bifunctional initiator, 2,2'-octamethylene bis-1,3-dioxolenium perchlorate. Chain scission by sodium and $n$-butyl alcohol proved the presence of the living di-cation. The deviation from monodisperse molecular weight distribution is explained by the rate of the initiation reaction.

KEY WORDS Tetrahydrofuran / Block Copolymer / Cationic Polymerization / Polytetrahydrofuran
\end{abstract}

The preparation of block copolymer by using the living nature of the cationic polymerization of tetrahydrofuran (THF) has been reported by Berger, et al., ${ }^{1}$ Saegusa, et al., ${ }^{2}$ and Lambert, et $a l^{3}$ They polymerized 3,3-bis(chloromethyl)oxetane $^{2}$ and thietane ${ }^{3}$ successively from polyTHF cation, or they reacted polystyrene anion ${ }^{1}$ with poly-THF cation. However, because of the low $T_{\mathrm{m}}\left(34^{\circ} \mathrm{C}\right)$ and $T_{\mathrm{g}}\left(-79^{\circ} \mathrm{C}\right)$ value of poly-THF, "ABA" type block copolymer in which soft segment $B$ consists of poly-THF seems most interesting and preparation of polyTHF dication may be desirable.

For this purpose, five types of initiators can be considered: (1) bis-carbonium salt, (2) Lewis acid plus promoter, (3) bis-oxonium salt, (4) bisacylcarbonium salt, and (5) bis-carboxonium salt.

Carbonium salt, such as triphenylmethyl hexachloroantimonate $^{4-6}$ or $p$-chlorobenzene diazonium hexaffuorophosphinate ${ }^{7}$ was shown to initiate the polymerization of THF by abstraction of hydrogen to form triphenylmethane or chlorobenzene, and bis-carbonium salt, $\mathrm{SbCl}_{6}{ }^{-}$. $\mathrm{Ph}_{2} \mathrm{C}^{+} \longrightarrow-\mathrm{CH}_{2} \mathrm{CH}_{2}-\Longrightarrow-\mathrm{C}^{+} \mathrm{Ph}_{2} \cdot \mathrm{SbCl}_{6}^{-}$ could not produce poly-THF di-cation. ${ }^{8}$ Although the mechanism of the initiation reaction changes from hydrogen abstraction to bonding in the propylene oxide-promoted polymerization by triphenylmethyl hexachloroantimonate, ${ }^{9}$ the decomposition of the triphenylmethyl ether end group of poly-THF during the polymerization was reported. $\mathrm{Sims}^{10}$ proposed the formation of di-cation in the epichlorohydrin-promoted polymerization of THF with phosphorous pentafluoride, but the reaction seems more complex according to the established reaction scheme. ${ }^{11}$ Berger, et al., did not succeed in obtaining poly-THF di-cation from boron fluoride and bifunctional epoxides. ${ }^{1}$ The bis-oxonium salt seems most interesting, because initiation by bonding was proved in the polymerization of THF with triethyloxonium tetrafluoroborate labelled by ${ }^{14} \mathrm{C} .{ }^{12}$ However, attempts to prepare bis-oxonium salt by the reaction of alkylene dihalide with silver perchlorate or antimony pentachloride in the presence of THF gave only mixtures, which are difficult for purification. Acyl cation was extensively studied by Olah and coworkers, ${ }^{13}$ and shown to be an active catalyst for the polymerization of THF. ${ }^{14}$ However, in spite of the claim for the formation of the living polymer of THF, ${ }^{15}$ we obtained a considerable amount of the termination product from the sodium methoxide killed-reaction mixture of THF initiated by acetyl hexafluoro- 
antimonate, hexachloroantimonate, or perchlorate. Carboxonium salt, prepared by Meerwein, et al., ${ }^{16}$ was reported to be an active initiator for the polymerization of THF. The formation of poly-THF di-cation from this type of catalyst system is presented.

\section{EXPERIMENTAL}

\section{Syntheses of Catalysts}

2-Methyl-1,3-dioxolenium Perchlorate. An amount of $5.4 \mathrm{~g}$ of 2-bromoethyl acetate was added to $250 \mathrm{ml}$ of a dry nitromethane solution containing $6.7 \mathrm{~g}$ of silver perchlorate at room temperature, and after two hours stirring, the catalyst solution was filtered from precipitated silver bromide. The catalyst solution was evaporated under reduced pressure, and the hygroscopic crystalline solid was obtained after washing with dichloromethane to remove the excess silver perchlorate. Leaflets, soluble in nitromethane and sulfur dioxide.

2-Methyl-1,3-dioxolenium Tetrafluoroborate. Twenty-eight grams $(0.121 \mathrm{~mol})$ of 2-ethoxyethyl acetate was heated with $38 \mathrm{~g}(0.20 \mathrm{~mol})$ of triethyloxonium tetrafluoroborate at 65 to $70^{\circ} \mathrm{C}$, while ethyl ether was distilled out. The solid mass was washed repeatedly with cold dichloromethane. The hygroscopic salt is soluble in nitromethane and sulfur dioxide, and soluble with difficulty in dichloromethane and tetrahydrofuran.

2,2'-Octamethylene-bis-1,3-dioxolenium Perchlorate. Five grams of bis-2-bromoethyl sebacate was added to $50 \mathrm{~m} l$ of dry nitromethane solution containing $7.5 \mathrm{~g}$ of silver perchlorate at room temperature, and after two hours stirring, the catalyst solution was filtered from precipitated silver bromide. After evaporating under reduced pressure and washing with ether, hygroscopic white leaflets were obtained.

2,2'-Octametylene-bis-1,3-dioxolenium Tetrafluoroborate. Six grams of bis-2-ethoxyethyl sebacate (bp $185-187^{\circ} \mathrm{C}(1.4 \mathrm{~mm})$ ) was heated with $8 \mathrm{~g}$ of triethyloxonium tetrafluoroborate while distilling ethyl ether, and after washing the solid mass repeatedly with dichloromethane, the white leaflets remained.

The purity of the catalyst was checked by NMR spectrum in nitromethane or sulfur dioxide.
2-Methyl-1,3-dioxolenium salt showed two singlet peaks of area ratio of $4: 3$ at $4.68 \tau$ and $7.20 \tau$ which are assigned to methylene and methyl protons, respectively. Considering the methylene protons of triethyloxonium salt and ethyl ether at $5.28 \tau$ and $6.55 \tau$ respectively and also with the methyl protons of triethyloxonium salt and ethyl acetate at $8.41 \tau$ and $7.97 \tau$ respectively, the following resonance hybrid may be proposed as a possible structure of the dioxolenium salt.<smiles>CC[O+]1OCC(C)=[C+]1C</smiles>

Preparation of 2,2'-Octamethylene bis-1,3-dioxolenium Perchlorate in vacuum system. In a reaction flask, sebacoyl dichloride was mixed with 1.5 equivalents of ethylene bromohydrin. After half an hour, hydrogen chloride and excess ethylene bromohydrin was removed off under reduced pressure, and the produced ester was extracted with dichloromethane. The dichloromethane solution after transfered to the flask (see Figure 1) was evaporated and the residue was dissolved by adding nitromethane. This nitromethane solution of bis-2-bromoethyl seba-

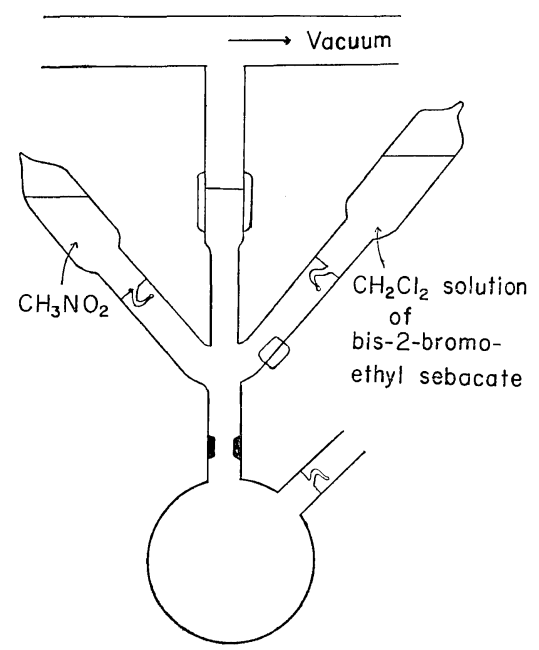

Figure 1. Apparatus for the preparation of bis-2bromoethyl sebacate in nitromethane. 


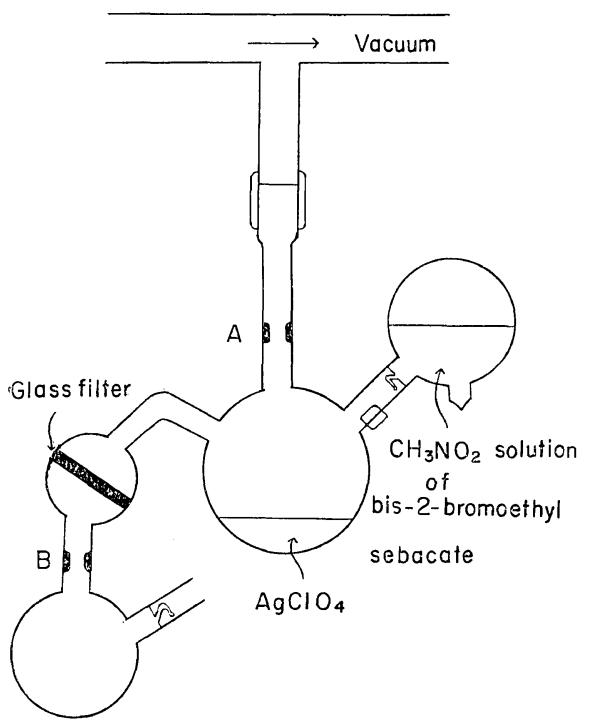

Figure 2. Apparatus for the preparation of 2,2'octamethylene bis-1,3-dioxolenium perchlorate.

cate is added through a breakable seal (see Figure 2) into 1.5 equivalent of silver perchlorate solution which was stored in high vacuum after sealing off at A. The yellow silver bromide precipitated from the homogeneous solution was filtrated through a glass filter and the tube was sealed at B. The nitromethane was evaporated and the excess silver perchlorate wa washed with ether to give a pure catalyst solid, which was redissolved in nitromethane, and the solu- tion was preserved at a low temperature. The NMR spectrum is shown in Figure 3, which can demonstrate the high purity of the catalyst.

Purification of Monomers and Solvents. THF was refluxed with potassium metal, and distilled from the blue colored solution of sodium naphthalene into an ampoule. Nitromethane was dried and distilled over calcium hydride.

\section{Polymerization Procedure}

A solution of the catalyst in nitromethane kept at $0^{\circ} \mathrm{C}$ was added to THF in a test tube under nitrogen. In the case of the bifunctional initiator, THF and the catalyst solution were mixed through a breakable seal in a vacuum system of $10^{-6} \mathrm{mmHg}$. The polymerization was terminated by adding diethylamine, and the poly-THF was precipitated by pouring into excess water, extracted with dichloromethane and after washing with water, the solvent was removed under reduced pressure and the poly-THF was freeze-dried from a benzene solution.

\section{Characterization of Poly-THF}

The number average molecular weight was measured by a Hitachi 115 vapor pressure osmometer or a Hewlett Packard 502 high speed membrane osmometer using benzene as a solvent.

The molecular weight distribution of polyTHF was measured by a Spinco Model E ultracentrifuge apparatus operating at a rotor speed $57780 \mathrm{rpm}$ at $0.2 \mathrm{~g} / \mathrm{d} l$ in ethyl acetate $-n$-hexane

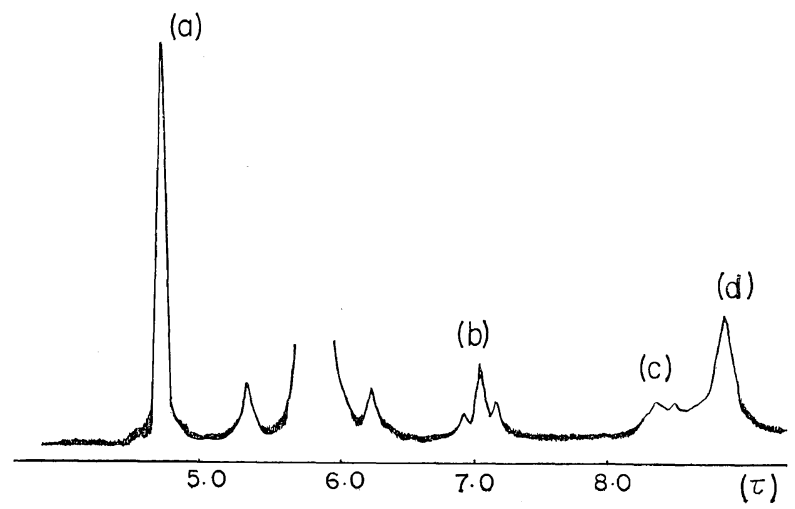

Figure 3. NMR spectrum of 2,2'-octamethylene bis-1,3-dioxolenium perchlorate in nitromethane

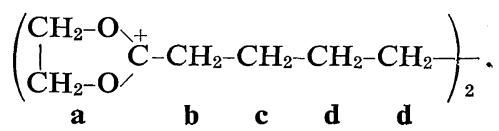




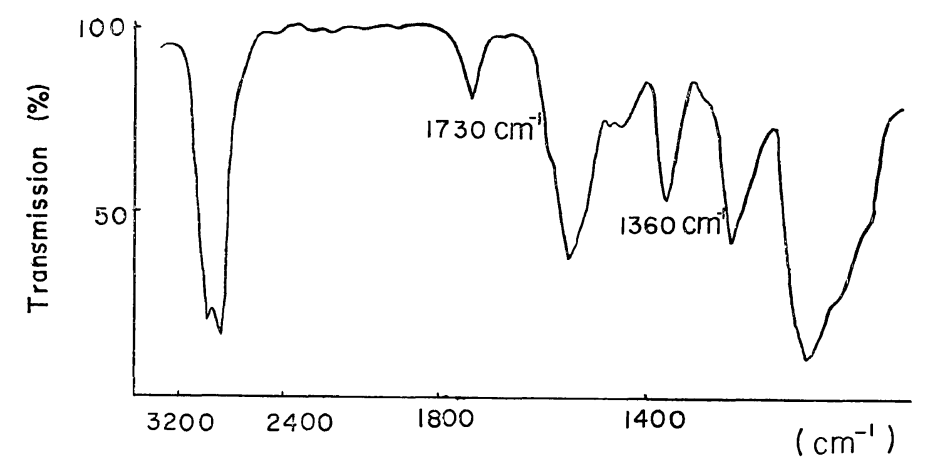

Figure 4. IR spectrum of poly-THF polymerized with 2-methyl-1,3-dioxolenium perchlorate.

mixture $(22.3 / 77.7 \mathrm{wt})$ at $30.4^{\circ} \mathrm{C}$ (theta solvent).

The acetate end group of poly-THF polymerized with 2-methyl-1,3-dioxolenium perchlorate was measured from the IR spectrum (Figure 4) in a carbon tetrachloride solution using absorption at $1730 \mathrm{~cm}^{-1}$ and $1360 \mathrm{~cm}^{-1}$ due to the ester group and methylene group, respectively, using a calibration curve obtained from a mixture of poly-THF polymerized with triethyloxonium tetrafluoroborate and 2-bromoethyl. acetate. The number of acetate end group per polymer chain was calculated from the degree of polymerization measured by a vapor pressure osmometer.

The scission experiment of poly-THF was carried out as follows: $0.5 \mathrm{~g}$ of poly-THF was dissolved in $100 \mathrm{~m} l$ of $n$-butyl alcohol, and refluxed for $48 \mathrm{hr}$ by adding $2 \mathrm{~g}$ of sodium metal. The reaction mixture was repeatedly washed with water to remove the catalyst fragment, and evaporated to dryness. The residue was dissolved in dichloromethane, washed with water, evaporated to dryness, and freeze-dried from a benzene solution.

\section{RESULT AND DISCUSSION}

Polymerization of THF with 2-Methyl-1,3-dioxolenium Perchlorate

The $\overline{\mathrm{DP}}$-conversion curve of poly-THF obtained with monofunctional carboxonium salt is shown in Figure 5. The straight line indicates the calculated $\overline{\mathrm{DP}}$ assuming rapid initiation without termination or chain transfer and also formation of one polymer chain from one catalyst molecule. The increase of $\overline{\mathrm{DP}}$ with conversion shows the living nature of this catalyst system, but $\overline{\mathrm{DP}}$ is more than twice that of the calculated value, approaching the calculated value at high conversion.

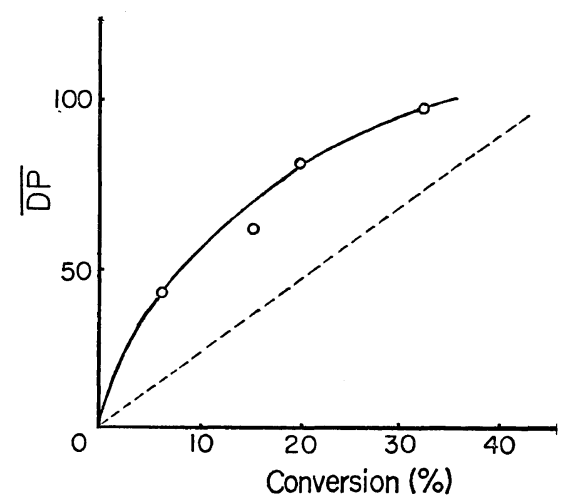

Figure 5. $\overline{\mathrm{DP}}$-conversion curve for the polymer1zation of THF with 2-methyl-1,3-dioxolenium perchlorate in nitromethane at $0^{\circ} \mathrm{C} ;[\mathrm{THF}]_{0}, 8.24 \mathrm{~mol} / l$; $[\mathrm{I}]_{0}, 5.65 \times 10^{-2} \mathrm{~mol} / l$.

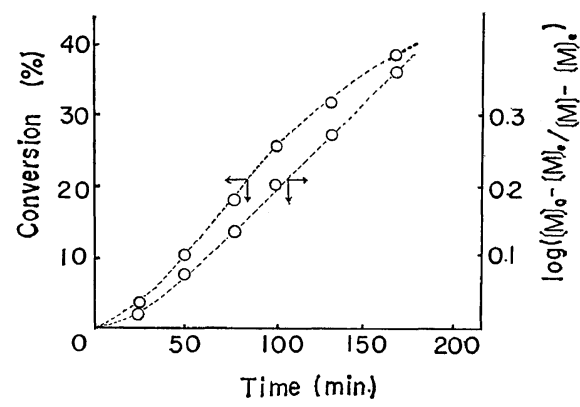

Figure 6. Time-conversion curve for the polymer1zation of THF with 2-methyl-1,3-dioxolenium perchlorate in nitromethane at $0^{\circ} \mathrm{C}$; $[\mathrm{THF}]_{0}, 8.24 \mathrm{~mol} / l$; $[\mathrm{I}]_{0}, 5.65 \times 10^{-2} \mathrm{~mol} / l$. 
This is explained by the slow rate of the initiation reaction. In Figure 6 , the time-conversion curve also shows the slow initiation and the first order plot of the rate of polymerization becomes linear after several minutes from the first. The apparent $K_{p}$ calculated from the first order rate of the polymerization and divided by the initial catalyst concentration is $1.65 \times$ $10^{-3} \mathrm{l} / \mathrm{mol} \mathrm{sec}$, is also considerably smaller than the case of triethyloxonium perchlorate: $4.10 \times$ $10^{-3} \mathrm{l} / \mathrm{mol} \mathrm{sec}$, or less than the usual absolute $K_{p}$ of THF: $5 \pm 1 \times 10^{-3} \mathrm{l} / \mathrm{mol} \mathrm{sec}$ at $0^{\circ} \mathrm{C}$ irrespectively of solvent and catalyst. ${ }^{17}$ Thus the initiation reaction is considerably slower than the propagation reaction, and only half of the catalyst seems active in the initial stage and all the

(b)

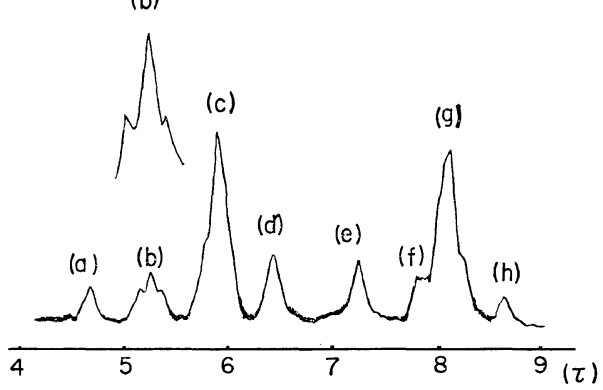

Figure 7. NMR spectrum of the reaction mixture of THF and 2-methyl-1,3-dioxolenium perchlorate in sulfur dioxide at $0^{\circ} \mathrm{C}$.

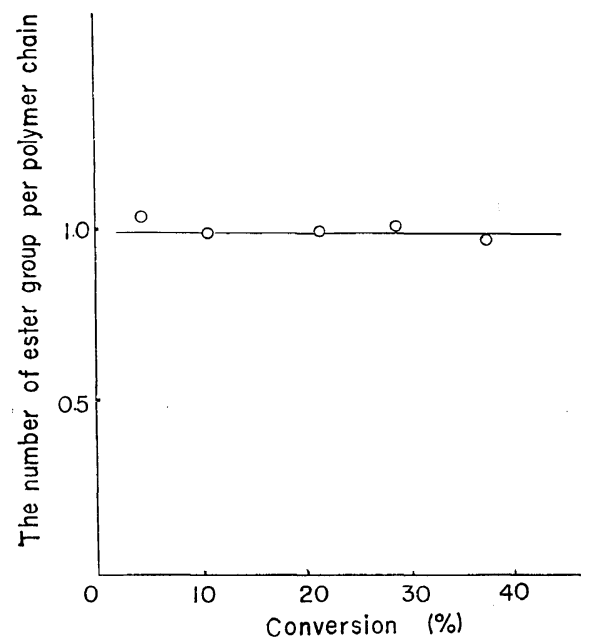

Figure 8. Number of terminal acetate group per polymer chain of poly-THF. catalyst is consumed only after a long reaction time.

The mechanism of the initiation reaction is clarified from Figures 7 and 8. The NMR spectrum of 2-methyl-1,3-dioxolenium perchlorate added to excess THF in sulfur dioxide is shown in Figure 7. This spectrum can be explained by assuming the following reaction.

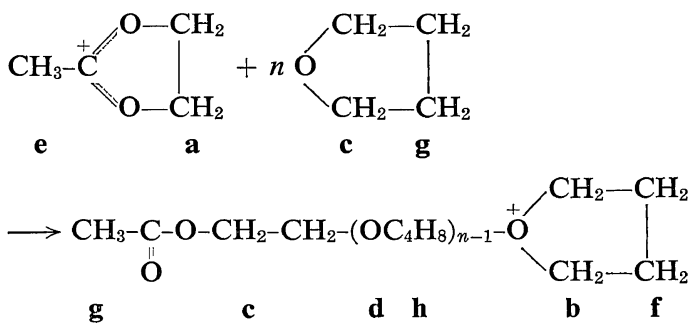

The decrease of a and e corresponds to the increase of $\mathbf{c}$ and $\mathbf{g}$, and $\mathbf{d}$ and $\mathbf{h}$ peaks come from poly-THF. $\quad b$ and $\mathbf{f}$ peaks can be assigned to the oxonium ion of poly-THF. Because of the slow initiation reaction, the NMR spectrum corresponding to<smiles>CC(=O)OCC[O+]1CCCC1</smiles>

could not be observed directly. Conclusive evidence for the bonding mechanism formulated in eq 1 was obtained from an IR spectrum of the derived poly-THF. The number of terminal acetate groups per polymer chain was unity from low conversion to high conversion, as shown in Figure 8.

Thus dioxolenium perchlorate is shown to be able to initiate the polymerization of THF by bonding and to facilitate the polymerization without termination or chain transfer. This is a prerequisite for any initiator suitable for the preparation of poly-THF di-cation. Attempted polymerization with 2-methyl-1,3-dioxolenium tetrafluoroborate could prove neither the living nature of the propagation reaction nor the bonding mechanism of the initiation reaction because of some unavoidable termination and chain transfer reaction.

Polymerization of THF with 2,2'-Octamethylene Bis-1,3-dioxolenium Perchlorate

Based on the above experiments, bifunctional 
dioxolenium salt was chosen as a suitable initiator to prepare poly-THF di-cation. 2,2'-Octamethylene bis-1,3-dioxolenium perchlorate was prepared from bis-2-bromoethyl sebacate and silver perchlorate in nitromethane under high vacuum to avoid the contamination of water. Polymerization of THF was also carried out in a high vacuum system by adding an aliquot of the catalyst solution. The results are shown in Table I and Figure 9. The increase of molecular weight with conversion suggests the living nature of this catalyst system. The characteristic slow rate of polymerization is due to the small $K_{p}$ value of THF, which is $5 \pm 1 \times 10^{-3} l / \mathrm{mol} \mathrm{sec}$ at $0^{\circ} \mathrm{C}$ in any solvent or catalyst. It takes ten days to reach $\overline{M_{n}}$ of one hundred thousand, and thirty days to reach $\overline{M_{n}}$ of three hundred thousand, irrespectively of the initiator concentration, because $\overline{\mathrm{DP}}$ is expressed by (Polymerized monomer concentration/initiator concentration) and polymerized monomer con-

Table I. Polymerization of THF with $2,2^{\prime}$-octamethylene bis-1,3-dioxolenium perchlorate in nitromethane at $0^{\circ} \mathrm{C}^{\mathrm{a}}$

\begin{tabular}{cccccc}
\hline \multirow{2}{*}{$\begin{array}{c}\text { Run } \\
\text { no. }\end{array}$} & $\begin{array}{c}\text { Time, } \\
\text { day }\end{array}$ & $\begin{array}{c}\text { Conver- } \\
\text { sion, } \%\end{array}$ & Obsd & Calcd & $\begin{array}{c}\text { After } \\
\text { scission }\end{array}$ \\
\hline $7-1$ & 14 & 8.54 & 17.3 & 11.2 & - \\
$7-2$ & 24 & 15.6 & 23.5 & 20.4 & 16.7 \\
$7-3$ & 31 & 19.6 & 27.3 & 25.6 & - \\
$7-4$ & 46 & 21.6 & 32.2 & 28.3 & 23.0 \\
\hline
\end{tabular}

a $[\mathrm{M}]_{0}, 11.7 \mathrm{~mol} / l ;[\mathrm{I}]_{0}, 6.45 \times 10^{-4} \mathrm{~mol} / l$.

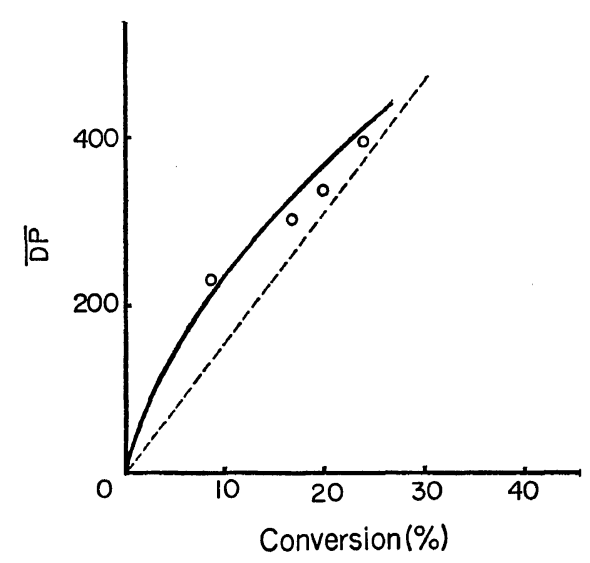

Figure 9. $\overline{\mathrm{DP}}$-conversion curve for the polymerization of THF with 2,2'-octamethylene-bis-1,3-dioxolenium perchlorate in nitromethane at $0^{\circ} \mathrm{C}$; $[\mathrm{THF}]_{0}, 11.7 \mathrm{~mol} / l ;[\mathrm{I}]_{0}, 6.45 \times 10^{-4} \mathrm{~mol} / l$.

centration is proportional to the initiator concentration and time of polymerization in a living polymerization.

Conclusive evidence for the formation of poly-THF di-cation is presented in the success of ion coupling with polystyrene di-anion to yield block copolymers of more than thirty repeating units of each segment. ${ }^{18}$ Partial evidence is presented by the scission experiment shown in the last column of Table $I$. By refluxing poly-THF with sodium and $n$-butyl alcohol, the molecular weight decreases to about two thirds of the initial polymer. The disappearance of the $\nu_{\mathrm{CO}}$ band at $1740 \mathrm{~cm}^{-1}$ in the IR spectrum of the product supports the following reaction scheme.

$$
\begin{aligned}
& \mathrm{HO}-\left(-\mathrm{C}_{4} \mathrm{H}_{8} \mathrm{O}-\right)_{m} \mathrm{CH}_{2}-\mathrm{CH}_{2}-\mathrm{O}-\underset{\|}{\mathrm{C}}-\left(-\mathrm{CH}_{2}-\right)_{\overline{8}} \mathrm{C}-\mathrm{O}-\mathrm{CH}_{2}-\mathrm{CH}_{2}-\left(-\mathrm{OC}_{4} \mathrm{H}_{8}-\right)_{n} \mathrm{OH} \\
& \stackrel{\mathrm{Na}, \mathrm{C}_{4} \mathrm{H}_{9} \mathrm{OH}}{\longrightarrow} \mathrm{HO}-\left(-\mathrm{C}_{4} \mathrm{H}_{8} \mathrm{O}-\right)_{\bar{m}} \mathrm{CH}_{2}-\mathrm{CH}_{2}-\mathrm{OH}+\mathrm{HO}-\left(-\mathrm{CH}_{2}-\right)_{10} \mathrm{OH}+\mathrm{HOCH}_{2} \mathrm{CH}_{2}-\left(-\mathrm{C}_{4} \mathrm{H}_{8} \mathrm{O}-\right)_{\bar{n}} \mathrm{OH}
\end{aligned}
$$

Although decrease to one half is expected theoretically, some initiator might have polymerized the THF by reacting with only one dioxolenium group, which might be supposed from the slow initiation compared to propagation as shown in Figure 9. The fraction $x$ of polymers propagated from both the dioxolenium groups of the initiator can be calculated from run $7-2$ and $7-4$ as $40 \%$,

$$
\begin{array}{ll}
16.7(1-x)+16.7(2 x)=23.5 & x=0.41 \\
23.0(1-x)+23.0(2 x)=32.2 & x=0.40
\end{array}
$$

and another $60 \%$ consists of a polymer propagated from one end. However, the experimental loss during the purification procedure of poly-THF and the experimental difficulty in determining 
Polymerization of THF by Bifunctional Initiator

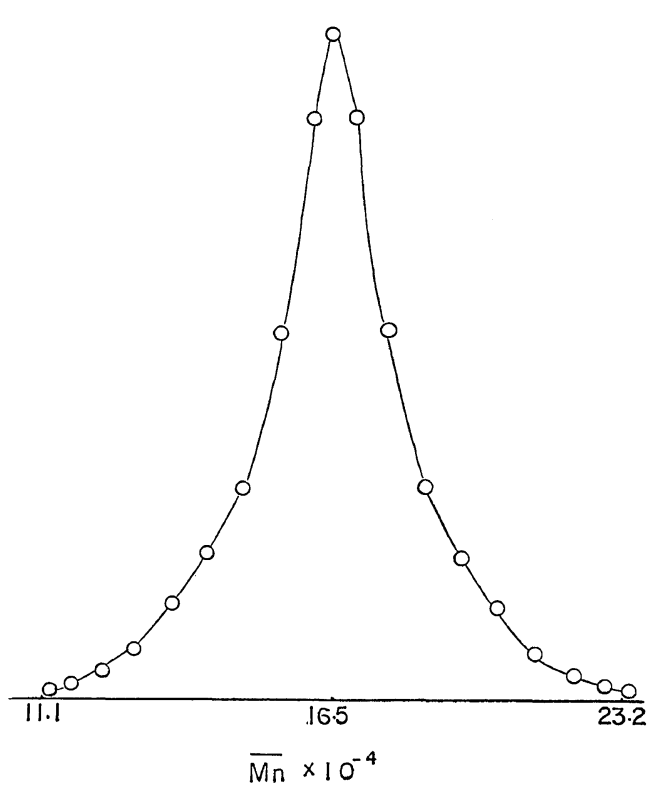

Figure 10. Molecular weight distribution curve of poly THF polymerized with $2,2^{\prime}$-octamethylene bis-1,3-dioxolenium perchlorate in nitromethane at $0^{\circ} \mathrm{C}$; $[\mathrm{THF}]_{0}, 11.7 \mathrm{~mol} / l ;[\mathrm{I}]_{0}, 6.16 \times 10^{-4} \mathrm{~mol} / l$; conversion, $8.97 \% ; \overline{M_{n}}, 16.5 \times 10^{4}$.

the molecular weight of poly-THF containing a low molecular weight fraction cause this interpretation to be less conclusive. Attempted scission of poly-THF polymerized with $2,2^{\prime}$ octamethylene bis-1,3-dioxolenium tetrafluoroborate failed to find the decrease of molecular weight, showing the absence of di-cation probably because of termination and chain transfer.

The molecular weight distribution of poly-THF di-cation is shown in Figure 10. The deviation from monodispersity is attributed to the slow initiation reaction. Attempts to obtain polyTHF di-cation of narrow molecular weight distribution by accelerating the initiation reaction using promotors lead to the formation of mono-cation by chain transfer. ${ }^{19}$

\section{REFERENCES}

1. G. Berger, M. Levy, and D. Vofsi, J. Polym. Sci., Part B, 4, 183 (1966).

2. T. Saegusa, S. Matsumoto, and Y. Hashimoto, Paper presented at the 18th annual meeting of the Society of Polymer Science, Japan, Kyoto, May, 1969.

3. J. C. Lambert and E. J. Goethals, Makromol. Chem., 133, 289 (1970).

4. I. Kuntz, J. Polym. Sci., Part B, 4, 427 (1966).

5. M.P. Dreyfuss, J. C. Westfahl, and P. Dreyfuss, Macromolecules, 1, 437 (1968).

6. W. M. Pasika, J. Polym. Sci., Part A-1, 7, 1489 (1969).

7. M. P. Dreyfuss and P. Dreyfuss, J. Polym. Sci., Part A-1, 4, 2179 (1966).

8. I. Kuntz, J. Polym. Sci., Part A-1, 5, 193 (1967).

9. I. Kuntz and M. Melchior, J. Polym. Sci., Part $A-1,7,1959$ (1969).

10. D. Sims, Makromol. Chem., 89, 245, (1966).

11. T. Saegusa, S. Matsumoto and T. Ueshima, Makromol. Chem., 105, 132 (1967).

12. D. Vofsi and A. V. Tobolsky, J. Polym. Sci., Part A, 3, 3261 (1965).

13. G. A. Olah, J. Amer. Chem. Soc., 84, 2733 (1962); 85, 1328 (1963).

14. H. Meerwein, D. Delfs, and H. Morshee, Angew. Chem., 72, 927 (1960).

15. E. B. Ludvig, B. A. Rosenberg, T. M. Zvereva, A. R. Gantmakher, and S. S. Medvedev, Vysokomol. Soedin., 7, 269 (1965).

16. H. Meerwein, K. Bodenbenner, and P. Borner, Liebig's Ann. Chem., 632, 38 (1960).

17. T. Saegusa and S. Matsumoto, Macromolecules, 1, 442 (1968).

18. Y. Yamashita, M. Hirota, K. Nobutoki, Y. Nakamura, A. Hirao, S. Kozawa, H. Matsui, G. Hattori, and M. Okada, J. Polym. Sci., Part B, 8, 481 (1970).

19. Y. Yamashita H. Matsui, G. Hattori, S. Kozawa, and M. Hirota, Makromol. Chem., in press. 